\title{
Mild Breakthrough Infection in a Healthcare Professional Working in the Isolation Area of a Hospital Designated for Treating COVID-19 Patients - Shaanxi Province, China, March, 2021
}

\author{
Chaofeng $\mathrm{Ma}^{1,{ }^{1, x}}$; Songtao $\mathrm{Xu}^{2,{ }^{2, x}}$; Yecheng $\mathrm{Yao}^{3}$; Pengbo $\mathrm{Yu}^{4}$; \\ You $\mathrm{Xu}^{5}$; Rui $\mathrm{Wu}^{1}$; Hailong Chen'; Xiaoping Dong ${ }^{2,6,7,8, *}$
}

\section{Summary}

What is already known about this topic?

Healthcare workers are at high risk of acquiring COVID-19 from occupational exposure to COVID-19 virus during their daily medical service work. Excellent infection prevention and control measures and adequate personal protective equipment (PPE) are essential to reduce the risk of hospital-acquired COVID-19.

\section{What is added by this report?}

On March 17, 2021, a female healthcare professional who already received both doses of the COVID-19 vaccination and was working in the isolation area of a designated COVID-19 hospital was diagnosed with COVID-19 in Xi'an city. Her exposure likely occurred five days before illness onset when she obtained nasopharyngeal and oropharyngeal swabs from the two imported cases that were identified as belonging to the B.1.1.7 lineage, the variant first detected in the United Kingdom.

What are the implications for public health practices?

Since the healthcare worker had been fully vaccinated and had mild symptomatology, it is considered a mild breakthrough infection. All vaccines are associated with breakthrough infections. In addition to rigorous adherence to infection prevention and control measures, use of adequate PPE, and using good clinical practices, the potential role of chronic upper respiratory infection in acquiring COVID-19 during medical procedures deserves further consideration.

The coronavirus disease 2019 (COVID-19) pandemic, caused by COVID-19 virus also known as severe acute respiratory syndrome coronavirus 2 (SARS-CoV-2), was declared by the World Health Organization (WHO) on March 11, 2020 (1-2). To date, more than 130 million confirmed cases and 2.8 million deaths have been reported worldwide for a crude case-fatality rate of $2.2 \%$. COVID-19 vaccines made with several technologies are currently being used in many countries. China has a national policy to provide COVID-19 vaccines free of charge, and by the end of March 2021, nearly one hundred million people have been vaccinated. However, China's overall population immunity is far from established, and healthcare workers at the frontline of the fight against COVID-19 are at high-risk of acquiring COVID-19 during medical procedures (3-4). In many countries, healthcare workers, together with elderly people, residents and personnel in long-term care facilities, social care personnel, and workers in essential public services are prioritized for COVID-19 vaccination. We report the first domestic COVID-19 breakthrough infection in China - a vaccinated healthcare professional working in the isolation ward of a designated COVID-19 hospital.

\section{INVESTIGATION AND FINDINGS}

On March 17, 2021, Xi'an Health Commission reported a domestic COVID-19 case. The patient was a 36-year-old female laboratorian working in a hospital designated for treatment of imported COVID-19 cases. That day she was febrile, with a temperature of $37.9^{\circ} \mathrm{C}$; she had a cough and headache. She reported no dyspnea or shortness of breath. Chest computed tomography (CT) revealed flocculent shadows in both lungs. Throat swabs and blood samples were immediately obtained. Throat swabs were positive by COVID-19-specific quantitative reverse-transcription polymerase chain reaction (qRT-PCR), with the cycle threshold $(C t)$ values of 34 for $\mathrm{N}$ gene and 35.27 for ORF1ab fragment (Figure 1). Rapid COVID-19 antibody tests were weakly positive for $\mathrm{IgG}$ and 
negative for IgM. She was diagnosed as a confirmed COVID-19 case (regular type).

An epidemiological investigation was immediately launched. She had been healthy without fever or other relevant abnormalities during the 14 days before starting work in the isolation area on March 4 . She had been vaccinated with inactivated COVID-19 vaccine (Beijing Institute of Biological Products Co. LTD) on December 30, 2020 and again on January 20, 2021. She and her 33 workmates were working in the isolation area for a 3-week period. She lived in the dormitory in an area separate from the isolation wards. She lived and shared a bathroom in the dormitory with one colleague. On March 2 and March 11, she tested negative for COVID-19 by qRT-PCR in the hospital's routine testing of staff. Her main work was obtaining throat swabs for COVID-19 cases in the isolation wards and conducting blood, urine, and feces testing in the isolation area's BSL-2 laboratory. She alternated every other day with another laboratorian for this work. Medical history review showed that she has chronic rhinitis and usually breathes by mouth when wearing a mask.

After diagnosis, she was immediately transferred to the isolation ward for medical treatment and observation. Her highest temperature was $37.2^{\circ} \mathrm{C}$, recorded on March 19. Her cough increased slightly. She described dry mouth and mildly decreased sense of smell. Rapid testing for COVID-19 virus antibody showed IgG positive and IgM weakly positive. Chemiluminescence assay for total COVID-19 virus antibody appeared strongly positive with a cutoff index (COI) of 1,368 (Figure 1). On March 20, her temperature was normal, and her sense of smell improved slightly. Viral qRT-PCR was positive for N gene $(C t$. 37) and negative for ORFlab. Her temperature remained normal and all other symptoms disappeared in the following days. Her oxygen saturation was never below $98 \%$ and she never required supplemental oxygen. The patient remained in the isolation hospital due to weakly positive qRTPCR. On April 9, she recovered and was discharged from the isolation ward.

Whole viral genome sequencing was conducted on an insolate from a throat swab specimen obtained on March 17. Due to the low viral load of the sample, sequences covering only $82 \%$ of the entire genome were obtainable. Sequence analysis revealed that the

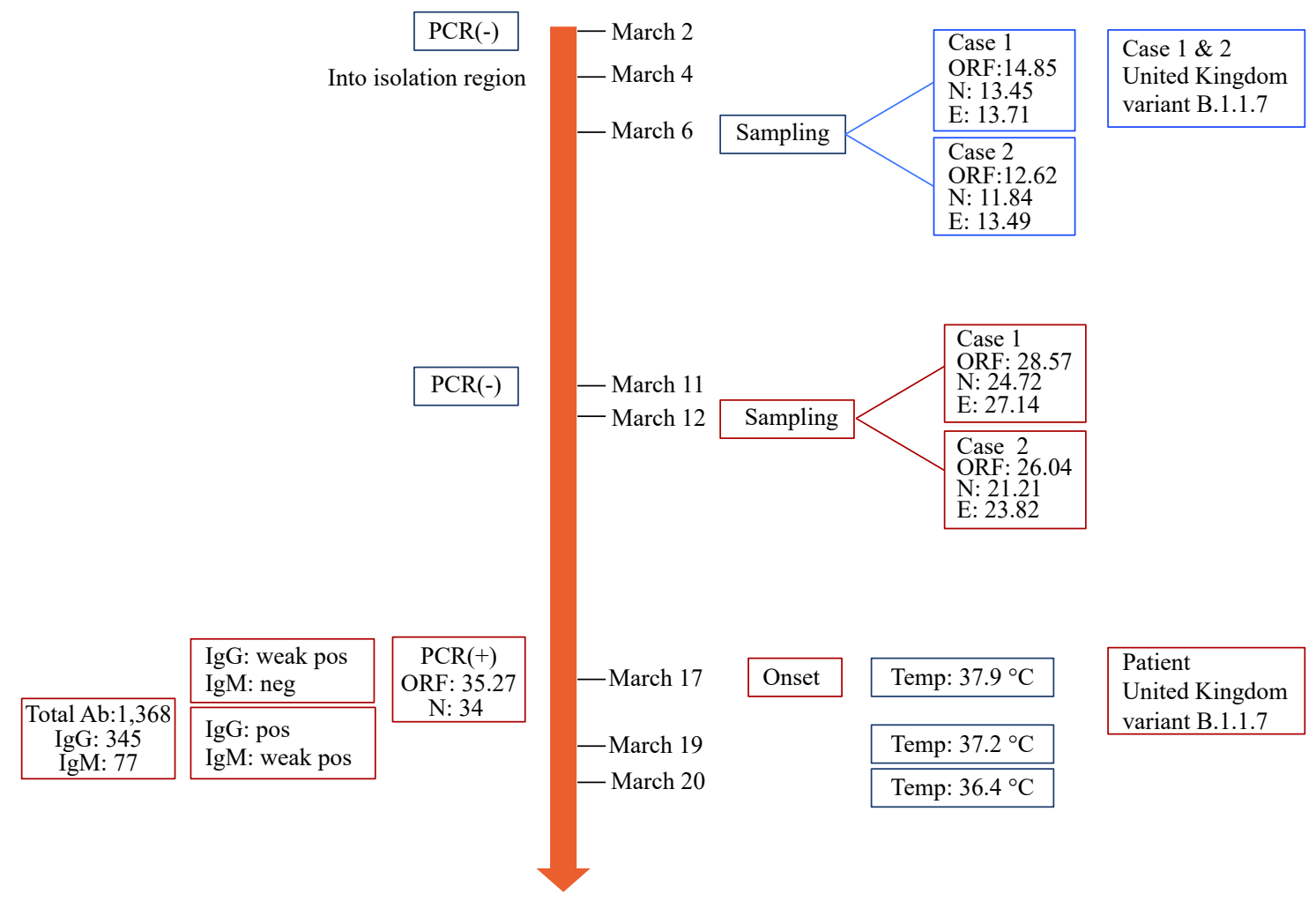

FIGURE 1. Timeline of the patient's medical work, her clinical course, and her virological testing.

Abbreviations: $P C R=$ polymerase chain reaction; Total $A b=$ Total antibody; IgG=Immunoglobulin $G$; IgM=immunoglobin $M$; ORF1ab=open reading frame $1 \mathrm{ab}$ gene; $\mathrm{N}=$ nucleocapsid gene; $\mathrm{E}=$ =nvelope gene; Temp=temperature; neg=negative; pos=positive. 
virus was a COVID-19 variant B.1.1.7 lineage virus and that it had high homology with isolates from 2 COVID-19 patients in the isolation ward who had returned to China from Uzbekistan on March 5.

The patient had two close contacts with both of the returnees from Uzbekistan. With assistance from other nurses, she obtained nasopharyngeal swabs (left and right nares) and an oropharyngeal swab from each returnee on March 6 and again on March 12. As shown in Figure 1, the 2 returnees had strongly positive qRT-PCR test results, with $C t$ values of the 3 targeted genes ranging from 11.84 to 14.85 in the March 6 samples and from 21.21 to 28.57 in the March 12 samples. During interviews with her and her colleagues, she indicated that she was well trained in use of personal protective equipment (PPE) and conducted medical procedures precisely, according to standard operating procedures.

The 33 colleagues on her team were transferred to another designated hospital for quarantine. Three serial qRT-PCR tests, conducted between March 17 and March 19, were all negative. None of the team members became febrile or ill. Prior to working in the isolation are, all 33 staff had been vaccinated with the same type of inactivated COVID-19 vaccine; 30 staff completed 2-dose regimens, while 3 received only 1 dose due to personal reasons. Blood samples from all 33 staff were obtained on March 19 and the levels of COVID-19 virus total antibody, IgG, and IgM were measured by chemiluminescence. As shown in Figure 2, all 30 fully vaccinated staff were positive in total antibody with an average COI of 19.77 (ranging from 1.7 to 150.83 ) and positive in $\mathrm{IgG}$ with an average COI of 20.29 (ranging from 3.69 to 64.33). The 3 staff who received 1 dose of vaccine tested negative $(\mathrm{COI}<1.0)$ in total antibody; 2 of the 3 were also $\operatorname{IgG}$ negative; 15 of the 30 fully vaccinated individuals were positive in IgM, while the 3 recipients that received 1-dose were negative. On Day 6, 13, 20, and 27 after quarantine, the 33 staff were tested again with qRT-PCR and all tested negative.

More than 3,000 healthcare workers and their family members were asked to stay at their work place or at home. All tested negative by qRT-PCR. Hundreds of environmental samples were obtained, including in the dormitory, isolation area, and other buildings of the hospital. Other than positive samples from the patient's room, all environmental samples were negative by qRT-PCR.

\section{DISCUSSION}

This case is the first infection of a healthcare professional working in an isolation region of a designated COVID-19 hospital since March 2020. Based on viral sequencing, this laboratorian clearly acquired infection from hospitalized, imported COVID-19 cases. There was no outbreak of COVID19 in Xi'an at the time, and the only domestic case reported since January was an imported case from Hebei Province who had been infected by a different strain of COVID-19 virus. Routine viral qRT-PCR testing before and 7 days after she entered the isolation area imply that it is extremely unlikely that she was infected outside of the isolation area. Due to a rapid and timely response, transmission into the community was prevented.

Based on the epidemiological investigation, we believe that the laboratorian was likely infected on March 12 when she obtained throat swabs for the 2 imported cases of COVID-19. She was negative by qRT-PCR on March 11, and on March 12, when she obtained swabs from the 2 returnees, both returnees had high viral loads of the B.1.1.7 variant. Simultaneously obtaining two nasopharyngeal swabs and one oropharyngeal swab is a high-risk procedure. People being sampled often sneeze or retch, expelling virus. The laboratorian's chronic rhinitis may have

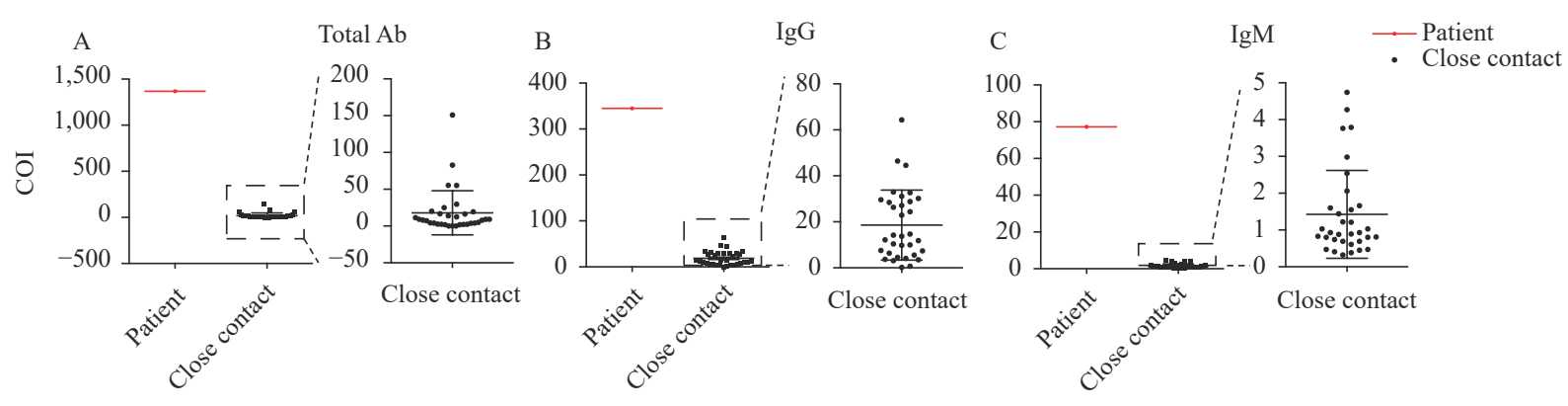

FIGURE 2. Serological assays for COVID-19 virus antibody for the patient and her 33 colleagues (shown as close contacts). Antibody titers were measured with commercial chemiluminescence kits for COVID-19 virus. Cutoff index (COI) values are indicated on the Y-axis. (A) Total antibody (Total Ab); (B) Immunoglobulin G (IgG); (C) Immunoglobin M (IgM). 
contributed to her propensity for mouth breathing, which may increase risk inhaling virus around a mask. Although the potential influence of chronic rhinitis on susceptibility to COVID-19 remains unknown, one study has showed that allergic rhinitis and asthma confer a greater risk of susceptibility to COVID-19 virus infection (5). We believe that a combination of factors may contribute to risk of infection.

The patient and 30 of her teammates had completed the full COVID-19 vaccination regimen by the beginning of February. Their serological assays confirmed COVID-19 virus seroconversion. Three individuals had received only one dose of vaccine; their serological tests showed low antibody levels. Unlike for the other staff, the patient's whole antibody, IgG, and IgM titers were all very high - an observation that implies that the patient had been immunized against by the vaccine, since the high titers are consistent with a boosting response. There are several types of COVID-19 vaccines on the market worldwide; all are effective, but none is $100 \%$ effective. One study showed lower efficacy in a high-risk population that had a high force of infection - healthcare professionals - compared with a community population (G). Our case report serves as a reminder of the importance of appropriate PPE for medical staff, even after vaccination. Globally-available COVID-19 vaccines have been demonstrated to prevent severe COVID-19 (7). Although it is not possible to prove by a single case study, the clinical course of the patient is consistent with a COVID-19 vaccination benefit making the illness mild and short.

The COVID-19 vaccines in use in China have very good efficacy, all meeting or exceeding the WHO Target Product Profile efficacy requirements. Breakthrough infections happen with all vaccines, and these breakthroughs merit study. We suggest conducting routine, systematic study of every fully vaccinated breakthrough case. Such study should include tracing the source of the virus, identifying the virus lineage to find variants, measuring antibody levels, and assessing for vaccine-associated enhancement of disease (VAED). Systematic study requires controls matched to breakthrough cases for assessing severity of illness (for VAED) and characteristics of the viruses and the vaccines (for effectiveness). Systematic study will help ensure that vaccination continues to be the safest and most effective way to prevent COVID-19.

Acknowledgements: Medical workers from Xi'an
No.8 Hospital and the staff of Shaanxi CDC and Xi'an CDC.

Funding: Chinese National Natural Science Foundation Grants (81630062), Grant (2019SKLID501) from the State Key Laboratory for Infectious Disease Prevention and Control, China CDC and Science Technology+Action Plan-Medical Research Project of Xi'an Science and Technology Bureau (2019115713YX012SF050).

doi: $10.46234 / \mathrm{ccdcw} 2021.094$

\# Corresponding author: Xiaoping Dong, dongxp238@sina.com.

\begin{abstract}
${ }^{1}$ Xi'an Center for Diseases Control and Prevention, Xi'an, Shaanxi, China; ${ }^{2}$ State Key Laboratory for Infectious Disease Prevention and Control, Collaborative Innovation Center for Diagnosis and Treatment of Infectious Diseases, National Institute for Viral Disease Control and Prevention, Chinese Center for Disease Control and Prevention, Beijing, China; ${ }^{3}$ National Institute for Nutrition and Health, Chinese Center for Disease Control and Prevention, Beijing, China; ${ }^{4}$ Shaanxi Center for Diseases Control and Prevention, Xi'an, Shaanxi, China; ${ }^{5}$ Xi'an No. 8 Hospital, Xi'an, Shaanxi, China; ${ }^{6}$ Center for Global Public Health, Chinese Center for Disease Control and Prevention, Beijing, China; ${ }^{7}$ Center for Biosafety Mega-Science, Chinese Academy of Sciences, Wuhan, Hubei, China; ${ }^{8}$ China Academy of Chinese Medical Sciences, Beijing, China.

\& Joint first authors.
\end{abstract}

Submitted: March 30, 2021; Accepted: April 07, 2021

\section{REFERENCES}

1. Zhu N, Zhang DY, Wang WL, Li XW, Yang B, Song JD, et al. A novel coronavirus from patients with pneumonia in China, 2019. N Engl J Med 2020;382(8):727 - 33. http://dx.doi.org/10.1056/NEJMoa2001 017 .

2. Di Gennaro F, Pizzol D, Marotta C, Antunes M, Racalbuto V, Veronese $\mathrm{N}$, et al. Coronavirus diseases (COVID-19) current status and future perspectives: a narrative review. Int J Environ Res Public Health 2020;17(8):2690. http://dx.doi.org/10.3390/ijerph17082690.

3. Rivett L, Sridhar S, Sparkes D, Routledge M, Jones NK, Forrest S, et al. Screening of healthcare workers for SARS-CoV-2 highlights the role of asymptomatic carriage in COVID-19 transmission. eLife 2020;9:e58728. http://dx.doi.org/10.7554/eLife.58728.

4. Gómez-Ochoa SA, Franco OH, Rojas LZ, Raguindin PF, Roa-Díaz ZM, Wyssmann BM, et al. COVID-19 in health-care workers: a living systematic review and meta-analysis of prevalence, risk factors, clinical characteristics, and outcomes. Am J Epidemiol 2021;190(1):161 - 75. http://dx.doi.org/10.1093/aje/kwaa191.

5. Yang JM, Koh HY, Moon SY, Yoo IK, Ha EK, You S, et al. Allergic disorders and susceptibility to and severity of COVID-19: a nationwide cohort study. J Allergy Clin Immunol 2020;146(4):790 - 8. http://dx. doi.org/10.1016/j.jaci.2020.08.008.

6. Palacios R, Patiño EG, de Oliveira Piorelli R, Conde M, Batista AP, Zeng G, et al. Double-blind, randomized, placebo-controlled phase III clinical trial to evaluate the efficacy and safety of treating healthcare professionals with the adsorbed COVID-19 (inactivated) vaccine manufactured by sinovac - PROFISCOV: a structured summary of a study protocol for a randomised controlled trial. Trials 2020;21(1):853. http://dx.doi.org/10.1186/s13063-020-04775-4.

7. Kashte S, Gulbake A, El-Amin III SF, Gupta A. COVID-19 vaccines: rapid development, implications, challenges and future prospects. Hum Cell 2021;7:1 - 23. http://dx.doi.org/10.1007/s13577-021-00512-4. 